\title{
Drug-Related Problems in Patients Undergoing Elective Total Joint Arthroplasty of the Hip or Knee
}

\author{
Melissa Haley, Colette Raymond, Cesilia Nishi, and Eric Bohm
}

\begin{abstract}
Background: Few studies have evaluated drug-related problems in patients undergoing elective total hip or knee arthroplasty.

Objective: To quantify, for patients undergoing elective total joint arthroplasty, drug-related problems arising from medication orders written before or immediately after the surgery. The primary outcome was the proportion of patients with at least one drug-related problem. The secondary outcomes were the total number and descriptions of these problems, according to the patient's age and the category, type, and severity of the drug-related problem.
\end{abstract}

Methods: From among patients who underwent elective total joint arthroplasty in a large Canadian regional health authority in 2005, 150 were randomly selected for this chart audit. Patients were included if they had been taking more than one medication before surgery. The charts were examined for drug-related problems, which were categorized according to whether the problem involved a prescription for a home medication, an order for a postoperative medication, or a potential indication for drug therapy. The problems were further described by type and potential severity.

Results: Of the 146 patients whose charts were available, 116 (79.5\%) had at least one drug-related problem, with a mean of 1.88 drug-related problems per patient. Of the 146 patients, $88(60.3 \%)$ had at least one drug-related problem involving a home medication, 34 (23.3\%) had problems related to postoperative orders, and $37(25.3 \%)$ had problems related to a potential indication. The mean number of drug-related problems per patient was 2.03 for those 65 years of age or older and 1.56 for those younger than 65 years $(p=0.09)$; however, more of the older patients experienced at least one drug-related problem related to home medications $(67 \%$ [67/100] versus $46 \%[21 / 46], p=0.02)$. The most common types of problems were medication omissions, illegible drug orders, inappropriate dose or frequency, and drug-allergy interactions. Of the 275 drug-related problems identified, 147 (53.5\%) were deemed potentially harmful, $78(28.4 \%)$ required monitoring, and $50(18.2 \%)$ were considered not harmful.

Conclusions: In this study, patients who underwent total joint arthroplasty experienced many drug-related problems. Pharmacists may have opportunities to optimize patient care by identifying, resolving, and preventing drug-related problems in this patient population.

Key words: total joint arthroplasty, drug-related problems

\section{RÉSUMÉ}

Contexte : Un nombre limité d'études ont évalué les problèmes reliés à la pharmacothérapie chez les patients devant subir une arthroplastie par prothèse totale de la hanche ou du genou.

Objectif : Quantifier, chez les patients devant subir une arthroplastie par prothèse totale non urgente, les problèmes reliés à la pharmacothérapie découlant des ordonnances de médicaments rédigées avant ou immédiatement après l'intervention. Le principal paramètre d'évaluation était la proportion de patients présentant au moins un problème relié à la pharmacothérapie. Les paramètres d'évaluation secondaires étaient le nombre total de ces problèmes et leurs descriptions, en fonction de l'âge du patient ainsi que de la catégorie, du type et de la gravité du problème relié à la pharmacothérapie.

Méthodes : Parmi les patients qui avaient subi une arthroplastie par prothèse totale non urgente au sein d'une importante régie régionale de la santé canadienne en 2005, 150 ont été choisis au hasard pour cet audit sur dossiers médicaux. Les patients étaient retenus aux fins de l'audit s'ils avaient pris plus d'un médicament avant l'intervention. Les dossiers médicaux ont été analysés à la recherche de problèmes reliés à la pharmacothérapie classés selon que le problème impliquait une ordonnance pour un médicament pris à la maison, une ordonnance pour un médicament postopératoire, ou une indication potentielle pour une pharmacothérapie. Les problèmes ont été classés plus finement par type et par gravité potentielle.

Résultats : Des 146 patients dont les dossiers médicaux étaient disponibles, $116(79,5 \%)$ présentaient au moins un problème relié à la pharmacothérapie, pour une moyenne de 1,88 problème relié à la pharmacothérapie par patient. Des 146 patients, 88 (60,3\%) présentaient au moins un problème relié à la pharmacothérapie impliquant un médicament pris à la maison, 34 (23,3\%) présentaient des problèmes reliés aux ordonnances postopératoires, et 37 (25,3\%) présentaient des problèmes reliés à une indication potentielle. Le nombre moyen de problèmes reliés à la pharmacothérapie par patient était de 2,03 pour les patients de 65 ans ou plus, et de 1,56 pour les patients de moins de 65 ans $(p=0,09)$; cependant, un plus grand nombre de patients plus âgés ont présenté au moins un problème relié à la pharmacothérapie impliquant un médicament pris à la maison $(67 \%$ [67/100] contre $46 \%[21 / 46], p=0,02)$. Les types de problèmes les plus courants étaient les omissions de médicaments, les ordonnances illisibles, les doses ou les fréquences d'administration inappropriées et les interactions médicament-allergie. Des 275 problèmes reliés à la pharmacothérapie recensés, 147 (53,5\%) ont été jugés potentiellement nocifs, 78 (28,4\%) ont nécessité une surveillance, et 50 (18,2\%) ont été jugés non nocifs. 
Conclusions : Les patients qui ont subi une arthroplastie par prothèse totale dans le cadre de cette étude présentaient de nombreux problèmes reliés à la pharmacothérapie. Les pharmaciens pourraient avoir des occasions d'optimiser les soins aux patients s'ils sont en mesure de repérer, de résoudre et de prévenir les problèmes reliés à la pharmacothérapie dans cette population de patients.

Mots clés : arthroplastie par prothèse totale, problèmes reliés à la pharmacothérapie

[Traduction par l'éditeur]

\section{INTRODUCTION}

$\mathrm{O}^{\prime}$ rthopedic patients often attend preadmission and prehabilitation clinics as part of their preparation for surgery. Preadmission clinics allow diagnostic tests to be scheduled and performed, preoperative assessments to be conducted, patient education to be provided, and discharge requirements to be identified on an outpatient basis. ${ }^{1-4}$ The goals of such clinics include preventing surgical complications and reducing the length of the hospital stay. ${ }^{3}$ Studies describing pharmacists' involvement in preadmission clinics have shown that such involvement can reduce the number of discrepancies in patients' medication histories. ${ }^{3,5}$

In contrast to preadmission clinics, prehabilitation clinics focus on patient care before the patient attends the preadmission clinic, providing an opportunity to optimize chronic medical conditions before surgery. Prehabilitation has been described as the enhancement of a patient's functional capacity, to enable the patient to withstand surgery. ${ }^{6}$ Optimization of a patient's medication therapy is one area where postoperative outcomes can be improved through preoperative assessment and management. ${ }^{7}$ It has been well established that the clinical activities of pharmacists improve patient care in the surgical setting ${ }^{1-5}$ and other patient care areas, ${ }^{8}$ which could include preadmission and prehabilitation.

Drug-related problems are events or circumstances involving drug therapy that actually or potentially interfere with desired health outcomes. ${ }^{910}$ The literature describing the prevalence of drug-related problems among patients undergoing preparation for surgery in preadmission clinics is limited. ${ }^{1-5}$

A prehabilitation clinic was recently created within the Winnipeg Regional Health Authority, a large Canadian regional health authority serving about 700000 people. This health region has 3 community hospitals that together account for about 3000 hip and knee total joint arthroplasty procedures annually. Professionals from several health care disciplines, including a pharmacist, work in the prehabilitation clinic. This study was undertaken to determine the prevalence of drug-related problems among patients undergoing elective arthroplasty before creation of the prehabilitation clinic and to characterize these problems by patient age and by category, type, and severity of the problems. The results of this study will be used to develop the role of the pharmacist in this area of practice.

\section{METHODS}

The medical records of patients who underwent elective total joint arthroplasty between January and December 2005 were examined retrospectively. This time period was selected to obtain a representative sample of patients who received standard care immediately before the prehabilitation clinic became operational. The study was limited to those who had undergone elective (rather than emergency) arthroplasty to ensure that all patients included in the study had undergone preoperative assessment at a preadmission clinic.

The study population was identified from the regional Joint Replacement Registry, a database of all patients who undergo total joint arthroplasty in the region. A convenience sample of 150 cases was randomly selected from the list of all elective, single total joint arthroplasty procedures performed at the 3 sites in the health region in 2005. The number of cases from each site was proportional to the total number of procedures performed at the site. The random number generator in Microsoft Excel was used in the random selection process. Patients were included if review of the medical chart revealed that they had been taking more than one medication at home before the surgery. Patients were excluded if the records from the preadmission clinic were incomplete or the medical records were unavailable.

The primary outcome was the proportion of patients with at least one drug-related problem arising from medication orders written before or immediately after the surgery. Secondary outcomes were the number and description of these problems according to patient age and the category, type, and severity of the problem.

The drug-related problems were grouped into 3 main categories: problems related to a home medication, problems related to a postoperative medication, and problems related to a potential indication for drug therapy. A problem was defined as being related to home medications if the patient had a 
postoperative prescription that was inconsistent with home medications. A problem was defined as being related to postoperative medications if the prescription for a postoperative medication (specifically antibiotics, analgesics, anticoagulants, or antiemetics) was potentially inappropriate. If a medication considered appropriate for a specific comorbid condition had not been prescribed (at home or in hospital) and the patient had no obvious contraindication to the drug, the problem was categorized as a potential indication for drug therapy.

The drug-related problems were then described according to the patients' age and the type and severity of the problems. The number of patients with at least one drug-related problem and the number of drug-related problems per patient were determined for patients younger than 65 years of age and those 65 years of age or older. The prespecified types of drug-related problems were drug-allergy interactions, therapeutic duplication, nonformulary drug, incorrect formulation, inappropriate dose or strength, inappropriate route of administration, inappropriate frequency, illegible order, omission of a medication, contraindication, or incorrect drug. ${ }^{11}$ This method of characterization was chosen to reflect drug-related problems that a hospital pharmacist would be likely to encounter at the point of order entry for home and postoperative medications. The severity of each problem was evaluated according to a condensed version of the index categorization system of the National Coordinating Council for Medication Error Reporting and Prevention: no harm, monitoring required, or harmful..$^{12}$ According to this categorization, a drug-related problem that was deemed to be harmful would, by definition, potentially cause the impairment of the physical, emotional, or psychological function or structure of the body..$^{12}$ Examples of this type of problem would be inappropriate dosing of a potentially toxic medication or prescription of a medication to which the patient is allergic. A

\section{Table 1. Baseline Characteristics 146 of Patients Admitted for Elective Total Joint Arthroplasty}

\begin{tabular}{lcl} 
Characteristic & No. (\%) & of Patients* \\
\hline Age, years, mean (range) & 68.5 & $(31-91)$ \\
\hline Sex & 62 & $(42.5)$ \\
Male & 84 & $(57.5)$ \\
Female & & \\
\hline Chronic medical conditions & 24 & $(16.4)$ \\
Coronary artery disease & 12 & $(8.2)$ \\
Congestive heart failure & 35 & $(24.0)$ \\
Diabetes mellitus & 6 & $(4.1)$ \\
Chronic obstructive pulmonary disease & & \\
Type of surgery & 60 & $(41.1)$ \\
Hip & 86 & $(59.0)$ \\
Knee & 6.2 & $(0-16)$ \\
\hline No. of home medications, & & \\
mean (range) & &
\end{tabular}

*Unless indicated otherwise. drug-related problem was considered to require monitoring if observation or recording of relevant physiological or psychological signs was needed. ${ }^{12}$ Examples of this type of problem would be prescription of a regular-release blood pressure medication in hospital for a patient who was taking a controlled-release formulation at home.

A licensed pharmacist (M.H.) reviewed all of the randomly selected medical charts to identify drug-related problems and to determine their category, type, and severity. A second pharmacist (C.R.) independently identified drug-related problems from a random selection of 15 charts $(10.3 \%$ of the total) to validate the process for identifying problems. The second pharmacist also assessed a random sample of 10 charts $(6.8 \%$ of the total) to validate the collection of data for each identified problem. Finally, all of the drug-related problems identified were reviewed by the second pharmacist (C.R.) for validation of the classification by category type and severity. Any discrepancies in the identification or classification of drug-related problems were resolved through discussion. The following sections of the chart were reviewed to identify drug-related problems: assessment forms from the preadmission clinic, which were completed by a preadmission nurse (containing a history of medications that the patient was taking before surgery); preoperative orders for postoperative administration of home medications; and orders for medications such as antibiotics and opioids to be administered perioperatively.

The data were collated into a database created in Microsoft Excel. The mean number of drug-related problems per patient and the proportion of patients with at least one drug-related problem were calculated. Differences between age groups (those younger than 65 years of age and those 65 years of age or older) were compared with Student $t$ tests and $\chi^{2}$ tests performed in Microsoft Excel.

The study protocol was approved by the University of Manitoba health research ethics board and the research impact committee of each of the 3 study institutions. Inclusion of patients in the Joint Replacement Registry was voluntary, and all information collected for this study was stored and used in a manner that would protect patient confidentiality.

\section{RESULTS}

\section{Prevalence of Drug-Related Problems}

Of the 150 patients in the convenience sample, charts could not be obtained for 4 (2.7\%). The mean age of the 146 patients for whom charts were reviewed was 68.5 years, and 84 $(57.5 \%)$ were women (Table 1). Of these 146 patients, 116 (79.5\%) had at least one drug-related problem, and a total of 275 such problems were identified (Table 2), for an overall mean of 1.88 drug-related problems per patient. Most of the drug-related problems were related to home medications (Table 


\section{Table 2. Prevalence of Drug-Related Problems (DRPs) by Category}

\begin{tabular}{lrlrl} 
Category of DRP & \multicolumn{3}{c}{$\begin{array}{c}\text { No. (\%) of Patients with } \mathbf{1} \\
\text { or More DRPs }(\boldsymbol{n}=\mathbf{1 4 6})\end{array}$} & \multicolumn{2}{c}{ No. (\%) of DRPs } \\
\hline All & 116 & $(79.5)$ & 275 & $(100)$ \\
Home medication & 88 & $(60.3)$ & 180 & $(65.5)$ \\
Postoperative medication & 34 & $(23.3)$ & 38 & $(13.8)$ \\
Potential indication & 37 & $(25.3)$ & 57 & $(20.7)$ \\
\hline
\end{tabular}

2). Only $30(20.5 \%)$ of the patients had no drug-related problems, whereas $48(32.9 \%)$ had a single drug-related problem, 25 (17.1\%) had 2 problems, and $16(11.0 \%)$ had 3 problems. Twenty-seven patients $(18.5 \%)$ had 4 or more drug-related problems.

\section{Drug-Related Problems According to Patient's Age}

Among patients at least 65 years of age $(n=100)$ and younger than 65 years of age $(n=46)$, the proportions who experienced at least one drug-related problem of any type were similar $(81 \%$ [81/100] versus $76 \%$ [35/46], $p=0.64)$. There was no statistically significant difference in the mean number of drug-related problems per patient between the 2 age groups (2.03 versus $1.56, p=0.09$ ). However, more of the older patients experienced at least one problem related to home medications (67\% [67/100] versus $46 \%[21 / 46], p=0.02)$. Similar proportions of patients in each age group experienced at least one problem related to a potential indication for therapy $(29 \%[29 / 100]$ versus $17 \%[8 / 46], p=0.25)$ or to postoperative medications (18\% [18/100] versus $35 \%$ $[16 / 46], p=0.61)$.

\section{Types of Drug-Related Problems}

The most common type of problem related to home medications was omission of a medication (32.8\% [59/180]), followed by illegible orders (16.7\% [30/180]) (Tables 3 and 4). The most common type of problem related to postoperative medications was inappropriate frequency (45\% [17/38]), followed by drug-allergy interaction (37\% [14/38]). A total of 57 problems were related to potential indications for drug therapy; this category included patients with a diagnosis of congestive heart failure or diabetes mellitus (and no obvious contraindications) for whom angiotensin-converting enzyme inhibitor had not been prescribed.

\section{Table 3. Examples of Drug-Related Problems by Category and Type}

DRP Category and Type

Home medication

Therapeutic duplication

Incorrect formulation

Inappropriate dose or strength

Inappropriate route

Inappropriate frequency

Omission of medication

Incorrect drug

Contraindication

Other

\section{Postoperative medication}

Drug-allergy interaction

Therapeutic duplication

Inappropriate dose or strength

Inappropriate frequency

Omission of medication

Contraindication

Potential indication

for drug therapy

\section{Example}

Prescriptions for both losartan and candesartan

Prescription for nifedipine regular release when $\mathrm{XL}$ (extended release) formulation was intended

Prescription for metoprolol $5 \mathrm{mg}$ when dose at home was $50 \mathrm{mg}$

Prescription for brimonidine eyedrops to be administered to both eyes when home prescription was for left eye only

Prescription for metoprolol regular release to be given once daily instead of twice daily

Patient was taking ramipril at home but had no prescription for this drug while in hospital

Patient was taking formoterol at home but had a prescription for salmeterol in hospital

Prescriptions for ramipril and spironolactone with high potassium obtained during preadmission clinic, with no serum electrolytes ordered on hospital admission

Allergy assessment required (morphine allergy recorded in chart as "nausea and vomiting")

Prescription for morphine for a patient with documented allergy to codeine

Prescription for both warfarin $5 \mathrm{mg}$ daily and warfarin $7.5 \mathrm{mg}$ daily

Lorazepam 5 to $10 \mathrm{mg}$ prescribed instead of intended dose of 0.5 to $1 \mathrm{mg}$

Prescription for lorazepam "as needed", with no dosing frequency specified

Order for preoperative antibiotic missing

Order for postoperative thromboprophylaxis missing

Celecoxib prescribed for patient with recent history of bleeding ulcer

Patient with myocardial infarction but no prescription for ß-blocker

Patient with diagnosis of diabetes mellitus but no prescription for angiotensin-converting

enzyme inhibitor

Patient with diagnosis of coronary artery disease but no prescription for statin 
Table 4. Number of Drug-Related Problems (DRPs) by Type and Category

\begin{tabular}{|c|c|c|c|}
\hline \multirow[b]{2}{*}{ Type of Drug-Related Problem } & \multicolumn{3}{|c|}{ DRP Category; No. (\%) of DRPs } \\
\hline & $\begin{array}{l}\text { Home Medications } \\
\quad(n=180)\end{array}$ & $\begin{array}{l}\text { Postoperative Medications } \\
\qquad(n=38)\end{array}$ & $\begin{array}{l}\text { Potential Indication } \\
\quad(n=57)\end{array}$ \\
\hline Drug-allergy interaction & 0 & $14(37)$ & 0 \\
\hline Therapeutic duplication & $4(2.2)$ & $2(5)$ & 0 \\
\hline Nonformulary drug & $10(5.6)$ & 0 & 0 \\
\hline Incorrect formulation & $6(3.3)$ & 0 & 0 \\
\hline Inappropriate dose or strength & $24(13.3)$ & $1(3)$ & 0 \\
\hline Inappropriate route of administration & $1(0.6)$ & 0 & 0 \\
\hline Inappropriate frequency & $22(12.2)$ & $17(45)$ & 0 \\
\hline Illegible order & $30(16.7)$ & $1(3)$ & 0 \\
\hline Omission of medication & $59(32.8)$ & $2(5)$ & 0 \\
\hline Contraindication & $11(6.1)$ & $1(3)$ & 0 \\
\hline Incorrect drug & $3(1.7)$ & 0 & 0 \\
\hline Other & $10(5.6)$ & 0 & $57(100)^{*}$ \\
\hline
\end{tabular}

${ }^{\star}$ All drug-related problems of this type related to comorbid conditions identified in the chart for which the patient was not receiving recommended drug therapy and for which the patient had no contraindications. As such, these problems could not be categorized in the same manner as problems related to home or postoperative medications.

\section{Severity of Drug-Related Problems}

Of the 275 drug-related problems identified in this study, 147 (53.5\%) were deemed potentially harmful, 78 (28.4\%) were deemed to require monitoring, and $50(18.2 \%)$ were not considered harmful, according to the index categorization system of the National Coordinating Council for Medication Error Reporting and Prevention. ${ }^{12}$

Of the 180 problems related to home medications, 78 (43.3\%) were deemed potentially harmful, $54(30.0 \%)$ were deemed to require monitoring, and $48(26.7 \%)$ were not considered harmful. An example of a potentially harmful problem related to home medications was an incorrect dose of methotrexate ordered in hospital (i.e., different from the home dose). An example of a problem classified as requiring monitoring was an order for diltiazem regular-release in hospital for a patient who had been taking diltiazem sustainedrelease at home.

Of the 38 postoperative drug-related problems, 32 (84\%) were deemed potentially harmful and $4(11 \%)$ were deemed to require monitoring, with only $2(5 \%)$ considered not harmful. The potentially harmful problems related to postoperative medications included an order for lorazepam "as needed" with no maximum dose or frequency and an order for morphine for a patient with a documented codeine allergy. A postoperative drug-related problem that was deemed to require monitoring was an inappropriate dosing schedule for oxycodone controlled-release ( 4 times per day, instead of the appropriate twice-daily schedule).

Of the 57 potential indications for drug therapy identified in this study, 37 (65\%) were deemed potentially harmful and 20 (35\%) were deemed to require monitoring. One example of a potentially harmful problem of this type was lack of prescrip- tion for a ß-blocker for a patient with previous ischemic heart disease (and no obvious contraindications). One example of a problem of this type that was deemed to require monitoring was lack of prescription for a statin for a patient over 65 years of age with a diagnosis of diabetes mellitus.

\section{DISCUSSION}

In this review of a random sample of patients who underwent elective total joint arthroplasty in a large Canadian health region, the majority of patients had at least one drug-related problem. Most of the problems identified were related to home medications, and over half were deemed potentially harmful. Patients 65 years of age or older had more potentially harmful drug-related problems; they also had many problems involving a potential indication for drug therapy.

These findings suggest that when pharmacists are screening patients for drug-related problems, they should focus on prescriptions for home medications, patients 65 years of age or older, the total number of home medications for patients with specific comorbidities, and patients with comorbid conditions. These priorities will help pharmacists to have the greatest possible impact on patient care.

Although there are limited data evaluating drug-related problems in patients undergoing total joint arthroplasty, the findings of the current study are consistent with those of a study conducted at the University Health Network in Toronto, Ontario. ${ }^{5}$ In that earlier study, Kwan and others ${ }^{5}$ evaluated pharmacists' medication assessments in a surgical preadmission clinic. In particular, they compared drug-related problems between a standard group of patients (with no pharmacist involvement) and an intervention group (with pharmacist involvement). The majority of postoperative problems were 
related to home medications $(70.1 \%$ in the intervention arm and $78.6 \%$ in the standard care arm), and $37.2 \%$ of patients had at least one postoperative problem related to home medications. ${ }^{5}$ These findings are similar to the results in the current study, in which $65.5 \%$ of problems identified were related to home medications; however, a greater proportion of the patients in the current study (60.3\%) had at least one drugrelated problem involving home medications. This difference could be attributable to differences in the study designs, as the study by Kwan and others ${ }^{5}$ included communication between the health care team and the patient, which would have allowed for clarification of intentional and unintentional medication discrepancies. Alternatively, the classification of drug-related problems might have differed between the 2 studies, which might have accounted for the differing results.

Kwan and others ${ }^{5}$ found that the most common postoperative problem related to home medications was omission of medications (46.4\%), and the most common problems related to postoperative orders involved incorrect dose and frequency. Similarly, the most common types of drug-related problems in the current study were omission of medications, illegible drug orders, inappropriate dose frequencies, and drug-allergy interactions. Thus, a focus on preventing these specific types of medication errors could result in fewer drug-related problems and fewer consequences for the patient.

Cornish and others ${ }^{13}$ studied unintended discrepancies between the physician's medication orders on admission and a comprehensive medication history obtained at the time of hospital admission for patients admitted to general internal medicine clinical teaching units. They found that $53.6 \%$ of patients had at least one unintended discrepancy, and the most common error ( $46.4 \%$ of errors) was the omission of regularly used medications. In addition, $38.6 \%$ of the drug-related problems identified had the potential to cause moderate to severe discomfort or clinical deterioration (as determined by consensus among evaluators). Likewise, we found that $60.3 \%$ of patients had at least one problem relating to home medication orders, with $43.3 \%$ of these problems deemed potentially harmful.

The strengths of this study included random selection of patients from a representative sample of joint surgeries in an entire health region. Drug-related problems were identified by auditing the patients' charts according to the information that would be available to a pharmacist in a prehabilitation clinic (i.e., medication history, preadmission clinic assessment, and laboratory values). In addition, to increase the validity of the results and to minimize the subjective nature of the process of identifying drug-related problems, a second pharmacist confirmed the assessment of each problem.

Audits of patient records are subject to several limitations. One disadvantage of retrospective studies is the possibility of missing patient data. In addition, the accuracy of the medication history could not be verified, the rationale for the choice of medications prescribed could not be determined, drug allergies could not be clarified, and intentional and unintentional drug omissions could not be evaluated. Because of the retrospective nature of the study, the true prevalence of drug-related problems might have been underestimated or overestimated. The only information available for audit was the patient's medication history, the preadmission clinic assessment, and laboratory values. Thus, information gathered from the patient record was limited by what had been documented; the patient was not available to verify actual use of home medications, and management or control of disease could not necessarily be determined. In addition, the intent of the prescriber in stopping or continuing drug therapy was not always documented. Assumptions were made about drug allergies, the physician's intent, medication history documented in the preadmission clinic, patient-reported medication history, and drug-related problems involving indications for drug therapy. More specifically, the broadest possible drug allergy or intolerance was assumed to be correct. Errors of medication omission were assumed to be unintentional, unless specifically indicated otherwise or unless the intention was obvious from the chart. The most comprehensive medication history documented in the chart was assumed to be the most accurate history. Finally, the method for categorizing drug-related problems in this study differed from what is traditionally employed in pharmacy practice. ${ }^{10}$ This categorization, modified from an evaluation of a medication error classification system, ${ }^{11}$ was chosen because the drug-related problems were being evaluated retrospectively, from the perspective of a hospital pharmacist at the point of order entry, with limited clinical information available. Although this perspective for assessing drug-related problems is limited, pharmacists in this situation do spend time identifying and resolving such problems as they fill prescriptions for patients who have undergone total joint arthroplasty. Only a prospective study could use a broader categorization of drug-related problems.

Despite the assumptions inherent in a retrospective approach, many drug-related problems were identified in this population of patients undergoing total joint arthroplasty. Furthermore, the problems pertinent to disease management, including potential indications for drug therapy, were considered as drug-related problems, albeit potential rather than actual. When these problems were excluded from the analysis, the total number of problems observed was still 218 (1.49 per patient), and $73 \%$ of the patients experienced at least one problem. Although a second pharmacist confirmed the categorization used in this project, there was still potential for misclassification of the drug-related problems. In addition, alternative tools for classifying drug-related problems, other 
than the tool used here, are available, and using one of the other tools available might have yielded different results.

In addition to obtaining patients' medication histories, pharmacists perform a variety of tasks to prevent and resolve drug-related problems in the perioperative setting, including checking availability in hospital of medications that patients are taking at home and arranging for suitable alternatives if unavailable; recording the patient's medication history in the chart for hospital use; writing prescriptions for discharge medications before the patient is admitted; counselling the patient about home medications, pre- and post-operative medications, and administration times; offering general health promotion activities; and communicating with the patient's primary care physician by sending a referral form with recommendations when drug-related problems are identified.

In conclusion, many drug-related problems occur in patients undergoing total joint arthroplasty, and a great opportunity exists for pharmacists to intervene to resolve these actual and potential problems. The results of this study will be used to develop the role of the pharmacist in the preadmission and prehabilitation clinics of the Winnipeg Regional Health Authority.

\section{References}

1. Bhanji AA, Fallon KM, LeBlanc SP. Pharmacy involvement in a surgery preadmission program. Am J Hosp Pharm 1993;50(3):483-486.

2. Hick HL, Deady PE, Wright DJ, Silcock J. The impact of the pharmacist on an elective general surgery pre-admission clinic. Pharm World Sci 2001; 23(2):65-69.

3. Gulka M, Walker J, Thiessen B, Gaucher M. Pharmacist-reviewed medication histories in a surgical preassessment clinic. Can J Hosp Pharm 1996;49(1):29-31

4. Harris L, Walters PA, Costello C. Effect of pharmaceutical pre-assessment on post-operative interventions. Int J Pharm Pract 2001;9 Suppl:R32.

5. Kwan Y, Fernandes OA, Nagge JJ, Wong GG, Huh JH, Hurn DA, et al. Pharmacist medication assessments in a surgical preadmission clinic. Arch Intern Med 2007;167(10):1034-1040.

6. Ditmyer MM, Topp R, Pifer M. Prehabilitation in preparation for orthopaedic surgery. Orthop Nurs 2002;21(5):43-54.
7. Halaszynski TM, Juda R, Silverman DG. Optimizing postoperative outcomes with efficient preoperative assessment and management. Crit Care Med 2004;32(4 Suppl):S76-S86.

8. Information paper on pharmaceutical care. Ottawa (ON): Canadian Society of Hospital Pharmacists; [cited 2006 Mar 22]. Available from: http://www.cshp.ca/dms/dmsView/1 1 IP-PharmaCare.pdf. Membership required to access content.

9. PCNE classification for drug-related problems. Version 5.01. Pharmaceutical Care Network Europe; revised 2006 [cited 2007 Mar 8]. Available from: http://www.pcne.org

10. Hepler CD, Strand LM. Opportunities and responsibilities in pharmaceutical care. Am J Hosp Pharm 1990;47(3):533-543.

11. Bobb A, Gleason K, Husch M, Feinglass J, Yarnold PR, Noskin GA. The epidemiology of prescribing errors: the potential impact of computerized prescriber order entry. Arch Intern Med 2004;164(7):785-792.

12. National Coordinating Council for Medication Error Reporting and Prevention (NCC MERP). NCC MERP index for categorizing medication errors. Rockville (MD): The Council; 2001 [cited 2006 Nov 31]. Available from: http://www.nccmerp.org/medErrorCatIndex.html

13. Cornish PL, Knowles SR, Marchesano R, Tam V, Shadowitz S, Juurlink $\mathrm{DN}$, et al. Unintended medication discrepancies at the time of hospital admission. Arch Intern Med 2005;165(4):424-429.

Melissa Haley, BSCPharm, ACPR, is a Hospital Telepharmacist based in Torbay, Newfoundland and Labrador.

Colette Raymond, BScPharm, PharmD, MSc, ACPR, is a Staff Development and Practice Evaluation Pharmacist, Department of Pharmaceutical Services, Health Sciences Centre Hospital, Winnipeg, Manitoba.

Cesilia Nishi, BScPharm, ACPR, is a Doctor of Pharmacy student in the Faculty of Pharmaceutical Sciences, University of British Columbia, Vancouver, British Columbia.

Eric Bohm, BEng, MD, MSC, FRCSC, is an Assistant Professor in the Section of Orthopedics, University of Manitoba, and is affiliated with the Joint Replacement Group, Winnipeg Regional Health Authority, Winnipeg, Manitoba.

\section{Address correspondence to:}

Melissa Haley

7 Davalan Place

Torbay NL A1K OB7

e-mail: mhaley@northwest.ca 